A. Weisbecker, M. Burmester \& A. Schmidt (Hrsg.): Mensch und Computer 2015 Workshopband, Stuttgart: Oldenbourg Wissenschaftsverlag, 2015, S. 61-68.

\title{
Gaze Guiding zur Unterstützung der Bedienung technischer Systeme
}

\author{
Kathrin Bischof ${ }^{1}$, Benjamin Weyers ${ }^{2}$, Barbara Frank ${ }^{1}$, Annette Kluge ${ }^{1}$ \\ Lehrstuhl für Wirtschaftspsychologie, RuhrUniversität Bochum ${ }^{1}$ \\ Virtual Reality Group, RWTH Aachen University ${ }^{2}$
}

\section{Zusammenfassung}

Die Vermeidung von Bedienfehlern ist gerade in sicherheitskritischen Systemen von zentraler Bedeutung. Um das Wiedererinnern an einmal erlernte Fertigkeiten für das Bedienen und Steuern technischer Systeme zu erleichtern und damit Fehler zu vermeiden, werden sogenannte Refresher Interventionen eingesetzt. Hierbei handelt es sich bisher zumeist um aufwändige Simulations- oder Simulatortrainings, die bereits erlernte Fertigkeiten durch deren wiederholte Ausführung auffrischen und so in selten auftretenden kritischen Situationen korrekt abrufbar machen. Die vorliegende Arbeit zeigt wie das Ziel des Wiedererinnerns auch ohne Refresher in Form einer Gaze Guiding Komponente erreicht werden kann, die in eine visuelle Benutzerschnittstelle zur Bedienung des technischen Prozesses eingebettet wird und den Fertigkeitsabruf durch gezielte kontextabhängige Ein- und Überblendungen unterstützt. Die Wirkung dieses Konzepts wird zurzeit in einer größeren DFGgeförderten Studie untersucht.

\section{Einleitung und Motivation}

Gemäß der Theory of Disuse von Bjork und Bjork (1992; Bjork 2011) nimmt die Abrufstärke von einmal erlerntem Wissen oder Fertigkeiten nach einer längeren Phase des Nichtgebrauchs ab. Dies führt zu einer erschwerten Abrufbarkeit dieser Fertigkeit, was allgemeinhin als „Vergessen“ bezeichnet wird. Bei der Bedienung von automatisierten technischen Systemen wie auch bei der Prozesskontrolle ist die Wahrscheinlichkeit hoch, dass einmal erlernte Fertigkeiten über einen längeren Zeitraum nicht verwendet werden (Kluge et al. 2009). Ein solcher Fertigkeitsverlust kann in kritischen Situationen zu fehlerhaften Eingaben und Bedienfehlern und schlussendlich zu Unfällen führen (Onnasch et al. 2014; Parasuraman et al. 2000). Um dies zu verhindern, werden in der Praxis sog. Refreshertrainings durchgeführt. Diese dienen dazu ein Leistungsniveau wiederherzustellen, das am Ende eines Ersttrainings bereits vorhanden war und seit diesem die benötigten Fertigkeiten innerhalb eines bestimmten Zeitintervalls nicht wieder abgerufen wurden (Kluge 
\& Frank 2014). Häufig werden solche Trainings in Simulatoren durchgeführt, die das reale technische System nachbilden und das gezielte und gefahrlose Ausführen (Auffrischen) bestimmter Bedienprozesse ermöglichen (Kluge et al. 2009).

Durch den Einsatz visueller Mechanismen ist es möglich, den Blick und damit die Aufmerksamkeit eines Benutzers auf bestimmte Elemente einer Benutzeroberfläche zu lenken. Dies wird im Folgenden als Gaze Guiding bezeichnet. Für die visuelle Lenkung der Aufmerksamkeit eignen sich besonders Mechanismen, die präattentiv, also vor der eigentlichen bewussten Aufmerksamkeit wahrgenommen werden (Ware 2004). Beispiele hierfür wären die Form, Farbe, Bewegung oder räumliche Position eines Objektes sowie die Richtung, in die sich ein Objekt bewegt. Mit entscheidend dafür, ob ein Objekt präattentiv wahrgenommen wird, ist aber auch, wie stark sich dieses von anderen in seiner Umgebung unterscheidet und wie unterschiedlich die Umgebungsobjekte untereinander sind.

Die in diesem Artikel vorgestellte Forschungsarbeit stellt eine Vorarbeit dar, die die Untersuchung von Gaze Guiding zur Unterstützung des Fertigkeitsabrufs hinsichtlich deren Wirksamkeit in einer DFG geförderten Studie ermöglichen soll. Dazu wird im Folgenden eine softwaretechnische Implementierung vorgestellt, die einen kontextabhängigen Einsatz von Gaze Guiding zur Anwendung von Refresher Interventionen ermöglicht. Es werden Einund Überblendungen verwendet, die in Abhängigkeit des aktuellen Systemzustands eines Beispielsystems die jeweilige Bedienoberfläche anpassen. Hierzu beschreibt der folgende Abschnitt das implementierte Softwaremodul im Detail sowie dessen Verwendung im Simulationssystem AWASim (Kluge \& Frank 2014), welches die Steuerung eines Abwasseraufbereitungssystems simuliert und für die zuvor genannte Studie verwendet wird. Hierzu wurden bereits umfangreiche Vorstudien durchgeführt und veröffentlicht (Kluge \& Frank 2014). Anschließend wird ein Szenario vorgestellt, in dem das Gaze Guiding den Nutzer bei der Bedienung der Anlage unterstützt und bei Fehlbedienungen in den Bedienprozess eingreift. Der Artikel schließt mit einer Zusammenfassung und einem Ausblick auf zukünftige Arbeiten ab.

\section{Gaze Guiding Rahmenwerk}

Für die Verwendung von Gaze Guiding für den Fertigkeitsabruf wurde ein Rahmenwerk in Java entwickelt, das einen Satz von visuellen Mechanismen bereitstellt, um Elemente oder Bereiche einer visuellen Benutzeroberfläche (GUI) abhängig vom Zustand des zu steuernden Prozesses hervorhebt. So kann der Benutzer gezielt an den aufzufrischenden Steuerungsprozess erinnert werden, ohne dabei den Interaktionskontext verlassen zu müssen. Die Arten der Hervorhebung oder Überblendung wurden dabei aus der Forschung zur Aufmerksamkeitslenkung abgeleitet und als XML basierte Beschreibung dem Gaze Guiding Rahmenwerk zur Verfügung gestellt. Die für das Gaze Guiding notwendige Zustandsdetektion beruht auf einer Zustandsmodellierung, welche Systemzustände als Menge von Systemwerten beschreibt, wobei diese mit einem bestimmten Wertintervall oder Grenzwert assoziiert werden. Im Folgenden wird zunächst diese Modellierung von Systemzuständen beleuchtet, woraufhin in Abschnitt 2.2 die einzelnen Gaze Guiding 
Mechanismen beschrieben werden, die zurzeit implementiert sind. Abschnitt 2.3 präsentiert im Anschluss die Implementierung des Gaze Guiding Rahmenwerks in seiner Gesamtheit.

\subsection{Systemzustandsmodellierung}

Im Rahmen der vorgestellten Arbeit werden Systemzustände als Menge von Systemwerten beschrieben, welche mit Grenzwerten oder Intervallen assoziiert werden. Sei daher eine Menge von Systemzuständen $S$ angenommen, wobei jeder Zustand $s_{i}$ in $S$ ein Tupel von Werten $w_{j}$ ist, so dass jedes $w_{j}$ einen Systemwert des zu steuernden Systems repräsentiert und Elemente der Menge $W$ aller messbaren Systemwerte ist. Für jeden Zustand $s_{i}$ sei weiterhin eine Funktion $g_{i}$ definiert, die alle Element von $s_{i}$ jeweils einem Intervall $[n, m]$ zuordnet. Ein System befindet sich daher in einem Zustand $s_{i}$ genau dann, wenn für alle $w_{j}$ von $s_{i}$ gilt, dass der gemessene Systemwert $w_{j, t}$ zum Zeitpunkt $t$ im assoziierten Intervall von $w_{j}$ liegt, daher $n$ $<=w_{j, t}<=m$ gilt.

Für die Implementierung des Rahmenwerks wurde eine XML basierte Beschreibung entwickelt, welche die Modellierung dieser Zustände ermöglicht. Im Rahmen der vorgestellten Arbeit wird diese Zustandsmodellierung genutzt, um bei so beschriebenen Systemwertkonstellationen entsprechende Gaze Guiding Mechanismen in die Benutzerschnittstelle einzublenden. Daher sollten nicht nur Zustände modelliert werden, welche Normalbetriebszustände beschreiben, sondern auch Fehlerzustände bis hin zu kritischen Zuständen. Das entsprechende Matching von Systemzuständen zu Gaze Guiding Mechanismen wird detailiert im folgenden Abschnitt diskutiert. Dabei ist wichtig, dass in der XML Beschreibung IDs für Zustände spezifiziert werden, die entsprechend in der Beschreibung des Gaze Guiding Modells referenziert werden können.

Diese Art der Modellierung von Systemzuständen ermöglicht eine sehr flexible Beschreibung, ist allerdings weder notwendigerweise überschneidungsfrei, was einzelne Zustände angeht, noch vollständig bezüglich der Gesamtheit aller möglichen Zustände eines Systems. Dies ist zurzeit dem Modellierer überlassen. Zukünftige Arbeiten zielen darauf ab, solche Überschneidungen automatisch zu identifizieren sowie den Modellierer mit weiteren visuellen Werkzeugen bei der Beschreibung der Zustände zu unterstützen. Weiterhin zielen zukünftige Arbeiten darauf ab auch unscharfe Beschreibungen zuzulassen.

\subsection{Gaze Guiding Modellierung}

Zur Beschreibung des Gaze Guidings werden XML-Beschreibungen für die Art der Hervorhebungen sowie deren Referenz auf den entsprechenden Zustand für eine Einblendung von einem Experten erzeugt, welche auf einem XML-Schema basieren und validiert werden können. Die XML-basierte Beschreibung umfasst Angaben darüber, auf welchen Bereich der visuellen Benutzerschnittstelle eine oder mehrere dieser Hervorhebungen angewendet werden sollen sowie über die Eigenschaften hinsichtlich ihrer Erscheinung. Darunter fallen etwa die Form, die Farbe oder die Größe. Abhängig von der Hervorhebung können weitere Angaben gemacht werden, bei denen entweder aus einigen vorgegebenen Werten ein spezifischer ausgewählt wird, wie die Zeigerichtung eines Pfeils, 
oder beliebig festgesetzt werden kann, wie die Frequenz eines Blinkens. Einige Angaben sind dabei notwendig, für andere werden Default-Werte verwendet, wenn diese nicht angegeben werden. Alle Hervorhebungen, die gleichzeitig beim Eintreten eines Zustandes auftreten sollen, werden bei der Beschreibung in einem sogenannten Toolset zusammengefasst, welches jeweils mit einem entsprechenden Zustand aus der Zustandsmodellierung assoziiert wird. Die Hervorhebungen bleiben dabei so lange eingeblendet, bis der Benutzer eine Aktion auf der Benutzeroberfläche durchführt. Die Implementierung stellt zurzeit unter anderem die folgenden Einblendungen zur Verfügung, die in Abbildung 1 beispielhaft $\mathrm{zu}$ sehen sind. Die der Abbildung zugrunde liegende technische Applikation zur Steuerung einer Wasseraufbereitungsanlage wird in Abschnitt 3 im Detail vorgestellt.

- Transparente abgedunkelte Fläche mit freiem Bereich für das relevante Element

- Anzeigen einer textuellen Nachricht, auch mit Piktogrammen. So zeigt Abbildung 1(a) mit einem Piktogramm einer Ventilschaltfläche, dass sich der Hinweistext auf ein Ventil bezieht.

- Farbiger Rahmen als Umrandung eines Elements (b), auch blinkend

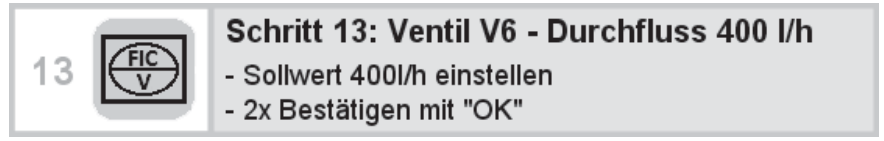

(a)

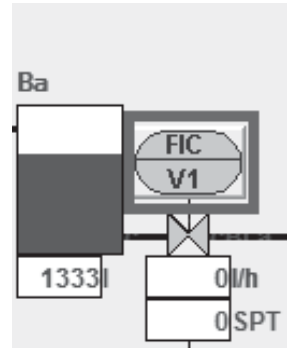

(b)

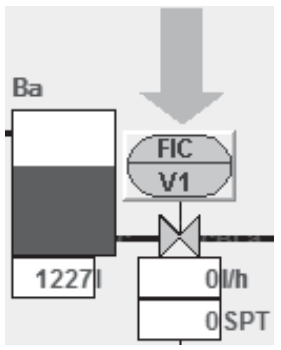

(c)

Abbildung 1: Beispiele für mögliche Einblendungen: (a) textuelle Nachricht mit einem Piktogramm eines Ventils, auf das sich die Nachricht bezieht (b) farbiger Rahmen, der die Schaltfläche des Ventils V1 umrandet (c) nach unten zeigender Pfeil auf die Ventilschaltfläche V1

- $\quad$ Ein Pfeil, der auf das entsprechende Element zeigt (c), auch blinkend

Darüber hinaus gibt es Hervorhebungen, die bis zum nächsten Eintreten eines Zustandes eingeblendet werden. Dazu gehört unter anderem ebenfalls ein farbiger Rahmen als Umrandung des relevanten Elementes und eine abgedunkelte transparente Fläche, die über einen ausgewählten Bereich gelegt werden kann. Des Weiteren kann angegeben werden, ob eine Linie zwischen zwei Einblendungen gezogen werden soll, um so beispielsweise eine visuelle Verbindung einer textuellen Nachricht mit einem Rahmen darzustellen, der den relevanten Bereich umgibt (Abbildung 2). 
Die Reihenfolge, in der die einzelnen Hervorhebungen in einem Toolset beschrieben werden, ist beliebig. Die Implementierung sieht vor, dass zuerst gegebenenfalls die abgedunkelte Fläche angezeigt wird, damit die anderen Einblendungen nicht durch diese verdeckt werden. Danach erscheinen, wenn angegeben, die Verbindungslinien gefolgt von den anderen Hervorhebungen.

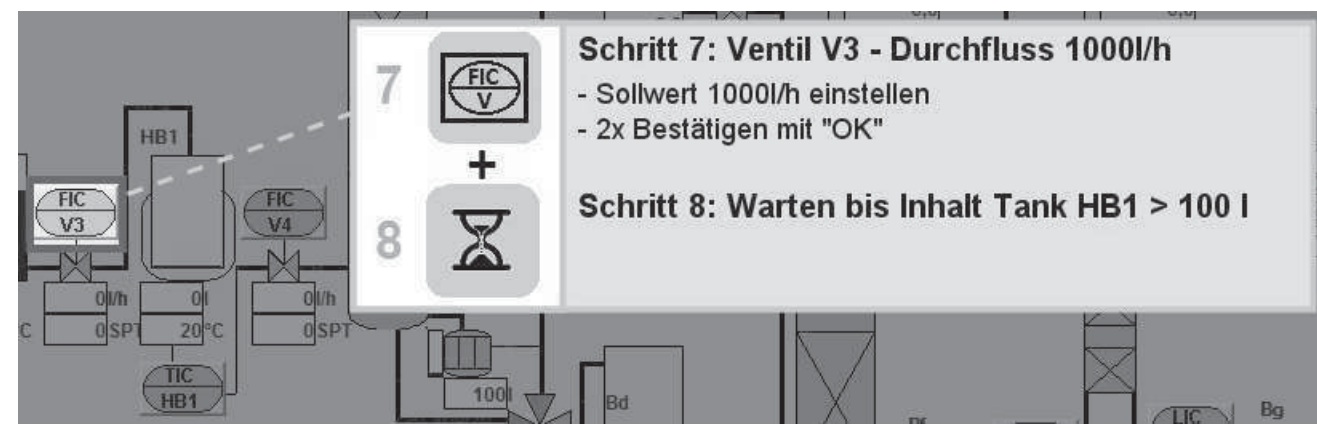

Abbildung 2: Beispiel einer Verbindungslinie zwischen einem Hilfstext mit zwei Piktogrammen und einem farbigen Rahmen, um anzuzeigen, dass sich die erste Anweisung im Hilfstext auf die Schaltfläche des Ventils V3 bezieht. Zudem wird eine abgedunkelte Fläche über die gesamte Benutzerschnittstelle gelegt, mit einem freien Bereich für die Schaltfläche des Ventils V3.

\subsection{Gaze Guiding Rahmenwerk - Implementierung}

Grundsätzlich kann das Rahmenwerkauf für jede Java Swing-basierte Benutzerschnittstelle angewandt werden. Der schematische Ablauf ist dabei in Abbildung $3 \mathrm{zu}$ sehen. Hierzu erfolgt die Einblendung auf das Fenster einer solchen Implementierung, so dass kein tiefgehender Eingriff in die zugrunde liegende Implementierung notwendig ist. Notwendig ist jedoch der Zugriff auf die aktuellen Systemwerte, was bspw. durch eine Extraktion von Systemwerten aus Logdaten ermöglicht werden kann.

Durch diesen Zugriff wird durch einen Regelauswerter geprüft, ob es ein Mapping zwischen den aktuellen Systemwerten und den Systemwerten eines beschriebenen Zustandes im Gaze Guiding Modul gibt (s. Abbildung 3, Mitte). Trifft dies zu, wird der so identifizierte Zustand über ein Matching mit seiner ID auf ein Toolset gematcht, sofern ein solches für den jeweiligen Zustand definiert wurde. Alle im Toolset enthaltenen Beschreibungen der Hervorhebungen werden dann in einem dritten Schritt mit der dazu passenden Methode auf der GUI in der obersten Ebene angezeigt, in diesem Fall der GUI von AWASim (s. Abbildung 3, Rechts). Dies erfolgt zum Beispiel durch das Zeichnen der Hervorhebung auf der GUI, das bloße sichtbar machen oder gegebenenfalls das Starten eines Threads für das Blinken. Die Einblendung des Toolsets erfolgt entweder so lange bis der Benutzer auf der Benutzeroberfläche eine Aktion durchführt oder bis ein anderer Zustand eintritt, da diese Hervorhebungen mit dem Zustand assoziiert sind. Bewirkt die Aktion des Benutzers allerdings keine Änderung des Zustandes, werden alle Hervorhebungen des Toolsets durch den beschriebenen Prozess nach einer bestimmten Zeitspanne, die ebenfalls gewählt werden kann, erneut eingeblendet. 
Wenn die aktuellen Systemwerte bereitgestellt werden, ermöglicht dieses Vorgehen Gaze Guiding zu verwenden, ohne das ein weiterer Zugriff auf den Quellcode des jeweiligen Systems notwendig ist. Dies wurde auch für die Durchführung der Studie verwendet.

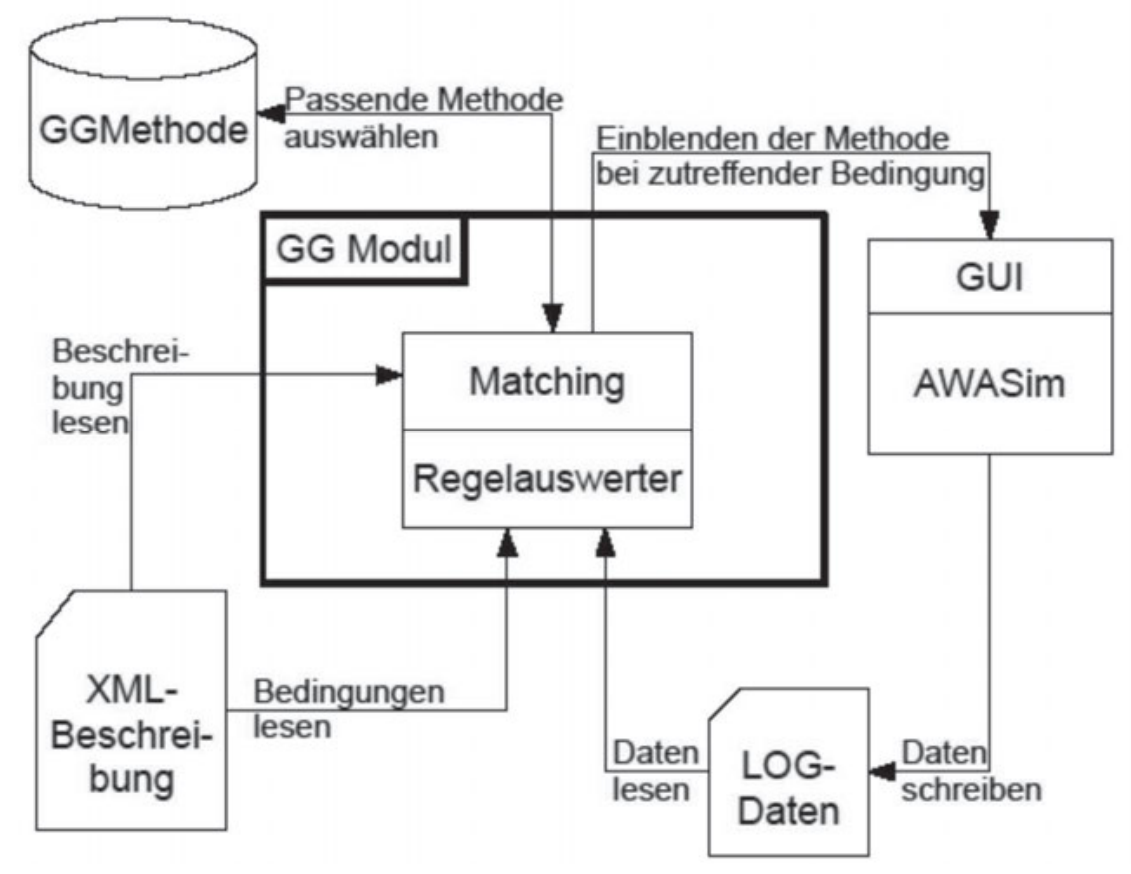

Abbildung 3: Schematischer Ablauf des Gaze Guidings

\section{Szenario}

AWASim (Kluge \& Frank 2014) ist eine Simulation für die Aufbereitung von industriellen Abwässern. Dabei wird ein Lösungsmittel-Wasser-Gemisch angeliefert, welches mit Hilfe der Abwasseraufbereitungsanlage in seine Bestandteile Wasser und Lösungsmittel aufgespalten wird, indem der Benutzer zunächst die Anlage durch die Ausführung bestimmter Bedienungsschritte anfahren und anschließend überwachen soll. Das Ziel bei der Simulation ist es, die Menge von gereinigtem Wasser zu maximieren und die Menge an anfallendem Abwasser möglichst gering zu halten. Einer dieser Schritte ist das Einschalten einer Heizbatterie (HB1), die dazu dient, das Lösungsmittel-Wasser-Gemisch auf eine bestimmte Temperatur zu erhitzen, sodass dies im nächsten Schritt in Wasser und Lösungsmittel getrennt werden kann. Bevor die Heizbatterie eingeschaltet wird ist zu beachten, dass der zugehörige Tank mit mindestens 100 Litern des Gemisches gefüllt ist, da sonst Schäden an der Heizbatterie entstehen können. Wird das Gemisch nicht ausreichend erhitzt, weil die Heizung nicht eingeschaltet wurde, kann es nicht getrennt werden und wird in einen Abfallbehälter umgeleitet. Um dies zu verhindern, können Zustände definiert 
werden, bei deren Eintreten durch das Gaze Guiding eine entsprechende Hervorhebung eingeblendet wird. Zum einen kann der Zustand für den Schritt vor dem Einschalten der Heizbatterie beschrieben werden und damit beim Eintreten dieses Zustandes eine Nachricht angezeigt werden, die den Nutzer darauf hinweist, dass die Batterie erst eingeschaltet werden soll, wenn der Tank mit 100 Litern des Gemisches gefüllt ist (siehe Abbildung 2). Wenn der erforderliche Füllstand und damit ein weiterer Zustand erreicht wird kann durch das Gaze Guiding ein weiterer Hinweis erscheinen, dass nun die Heizung eingeschaltet werden soll, wie in Abbildung 4 beispielhaft mit einem Hinweistext zu sehen ist. Zusätzlich werden hierbei zur weiteren Hervorhebung eine abgedunkelte transparente Fläche mit einem Ausschnitt für das relevante Element und ein roter Rahmen eingeblendet, um die Aufmerksamkeit des Benutzers auf die Schaltfläche für die Heizbatterie zu lenken.

Zum anderen kann als Reaktion auf eine fehlerhafte Eingabe dem Benutzer ein Hinweis angezeigt werden die Heizung einzuschalten, zum Beispiel wenn er schon den nächsten Schritt der Anfahrprozedur durchgeführt hat oder versucht, die Heizung zu früh einzuschalten. Der Auslöser für das Gaze Guiding wäre hierbei das Eintreten eines Zustandes, der eine verfrühte Ausführung eines anderen Bedienungsschritts repräsentiert.

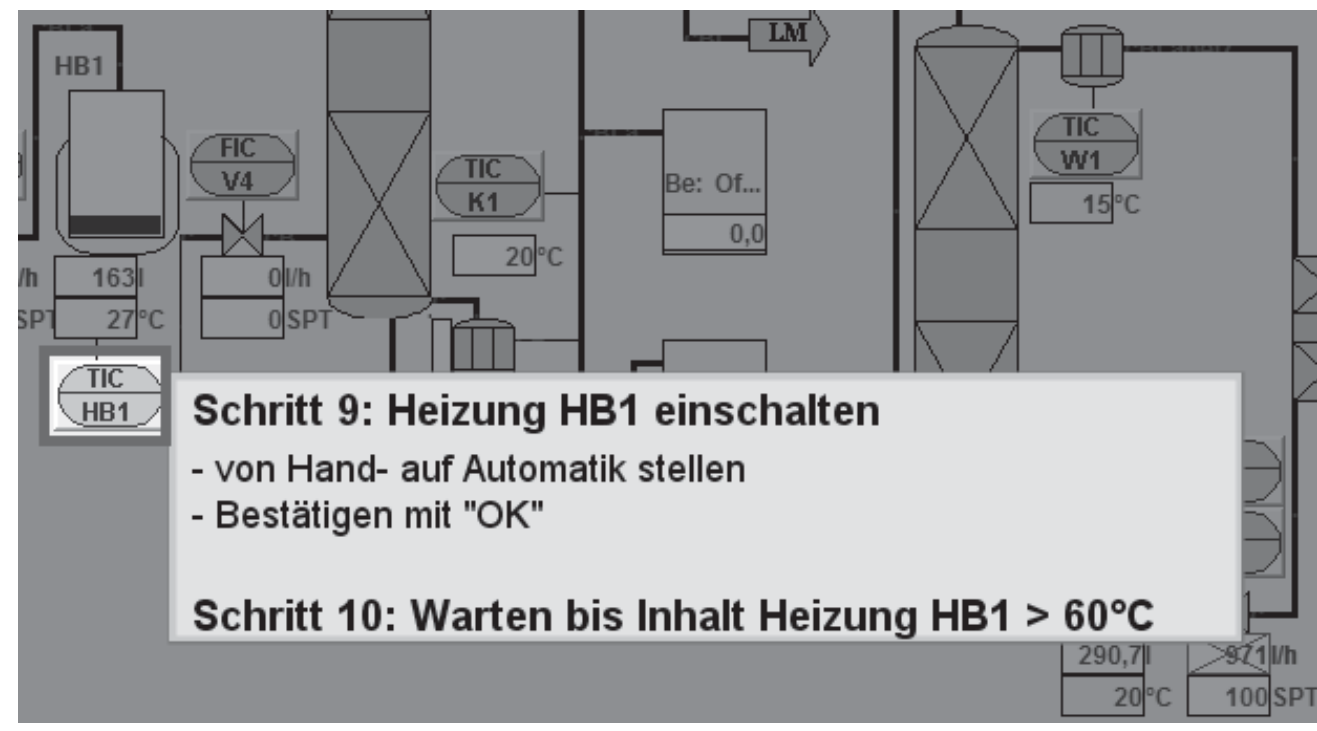

Abbildung 4: Hervorhebung der Schalfläche von HB1 mit Hinweistext, abgedunkelter Fläche und Rahmen.

\section{Zusammenfassung und Ausblick}

Im vorliegenden Artikel wurde ein Rahmenwerk vorgestellt, das abhängig von bestimmten Systemwerten Gaze Guiding Mechanismen einblendet, um dem Benutzer anzuzeigen, welcher Bedienungsschritt als nächstes vorzunehmen oder bei einer fehlerhaften Bedienung korrektiv zeitnah nachzuholen ist. Das Gaze Guiding Rahmenwerk wird derzeit im Rahmen einer von der DFG geförderten experimentellen Untersuchung als eine Alternative zu drei 
Refreshertrainings für die Prozesskontrollsimulation AWASim untersucht. In dieser Studie lernen die Teilnehmer/innen $\mathrm{zu}$ einem ersten Messzeitpunkt (t1) die Bedienung von AWASim. Nach einer Woche erfolgt ein Refreshertraining und nach einer weiteren Woche soll die gelernte Fertigkeit abgerufen werden ( $\mathrm{t} 2 ; 2 \times 5$ Between-Within-Subject Design). Die Refreshertrainings unterscheiden sich dabei in der Ausführung: Practice, hierbei wird die Simulation mit Hilfe eines Handbuchs angefahren, Skill Demonstration, bei der die Simulation nur einmal in einer Testsituation angefahren wird, und Symbolic Rehearsal, wobei die Teilnehmer/innen die Anfahrprozedur und die Bedienung theoretisch mit Hilfe von Fragebögen wiederholen. Darüber hinaus wird das Gaze Guiding eingesetzt, das anstelle eines Refreshertrainings zum letzten Messzeitpunkt (t2) eingesetzt wird. Eine weitere Gruppe, die Kontrollgruppe, erhält keine Unterstützung. Es soll vergleichend untersucht werden, inwieweit das Gaze Guiding ähnlich wirksam oder wirksamer ist, um den Fertigkeitsabruf zu unterstützen sowie Fehler zu reduzieren. Zudem wird untersucht, wie gut das Gaze Guiding den Mental Workload reduziert und wie sich dies auf die Situation Awareness auswirkt.

\section{Danksagung}

Das Forschungsprojekt „Die Wirkung von Refresher-Interventionen auf den Fertigkeitserhalt von komplexen, dynamischen Arbeitstätigkeiten der Prozesskontrolle über längere Zeitintervalle unter Berücksichtigung von Mental Workload und Situation Awareness“ wird unter dem Kennzeichen KL2207/3-3 durch die Deutsche Forschungsgemeinschaft (DFG) gefördert.

\section{Literaturverzeichnis}

Bjork, R. A. (2011). On the symbiosis of learning, remembering, and forgetting. In Benjamin, A. S. (Hrsg.): Successful remembering and successful forgetting: A Festschrift in honor of Robert A. Bjork. London, UK: Psychology Press, S. 1-22.

Bjork, R. A. \& Bjork, E. L. (1992). A new theory of disuse and an old theory of stimulus fluctuation. In Healy, A., Kosslyn, S. \& Shiffrin, R. (Hrsg.): From learning processes to cognitive processes: Essays in honor of William K. Estes (Vol. 2). Hillsdale, NJ, USA: Erlbaum, S. 35-67.

Kluge, A. \& Frank, B. (2014). Counteracting skill decay: Four refresher interventions and their effect on skill and knowledge retention in a simulated process control task. Ergonomics, 57(2), 175-190.

Kluge, A., Sauer, J., Schüler, K. \& Burkolter, D. (2009). Designing Training for process control simulators: a review of empirical findings and current practices. Theoretical Issues in Ergonomics Science, 10, 489-509.

Onnasch, L., Wickens, C. D., Li H., \& Manzey, D. (2014). Human Performance Consequences of Stages and Levels of Automation: An Integrated Meta-Analysis. Human Factors, 56(3), 476-488.

Parasuraman, R., Sheridan, T. B. \& Wickens, C. D. (2000). A Model for Types and Levels of Human Interaction with Automation. IEEE Transactions on Systems, Man, and Cybernetics- Part A: Systems and Humans, 30(3), 286-297.

Ware, C. (2004). Information Visualization: Perception for Design (2. Aufl.). Morgan Kaufmann. 